\title{
Pamidronate treatment for osteogenesis imperfecta in black South Africans
}

\author{
B D Henderson, ${ }^{1} \mathrm{MB}$ ChB, DCH, MMed; N Isaac, ${ }^{2}$ O Mabele, ${ }^{2}$ S Khiba, ${ }^{2}$ A Nkayi, ${ }^{2}$ T Mokoena ${ }^{2}$ \\ ${ }^{1}$ Division of Clinical Genetics, University of the Free State, and Universitas Hospital, Bloemfontein, South Africa \\ ${ }^{2}$ Undergraduate MB ChB students, Division of Clinical Genetics, University of the Free State, and Universitas Hospital, Bloemfontein, South Africa
}

Corresponding author: B D Henderson (gnmgbdh@ufs.ac.za)

\begin{abstract}
Background. Osteogenesis imperfecta is a heritable disorder of bone connective tissue. Type III has a high incidence in the black population of South Africa. Affected people experience numerous fractures, bone pain and progressive disability. Until the introduction of bisphosphonates to reduce fracture incidence, treatment revolved around orthopaedic and supportive care.

Objective. To assess the subjective attitude of patients towards pamidronate treatment.

Methods. Thirty black patients with osteogenesis imperfecta type III treated at Universitas Hospital were approached and 26 were included in this study. Patients or their parents were interviewed using a standardised researcher-administered questionnaire, either in person or by telephone.

Results. Most patients reported a reduction in symptoms, a feeling of increased wellbeing, increased strength and rated the pamidronate treatment highly. The intravenous route of administration and the side-effects experienced were bearable. Overall all patients would recommend this treatment to other affected persons.

Conclusion. This is first study to look at bisphosphonate treatment for osteogenesis imperfecta type III in black South Africans. The treatment is well tolerated and highly rated by the patients. Reported improvements and side-effects are similar to those reported in other populations. Using this form of treatment in this population is supported by these findings.
\end{abstract}

S Afr Med J 2016;106(6 Suppl 1):S47-S49. DOI:10.7196/SAMJ.2016.v106i6.10992

Osteogenesis imperfecta (OI) is a heritable disorder of the bone connective tissue characterised by defective bone connective tissue, bone fragility and decreased bone mass. ${ }^{[1,2]}$ Frequent bone fractures, reduction in bone mass, scoliosis, short stature, progressive long bone deformity and hearing loss are some of the features of this disease. ${ }^{[1]}$

Zeitlin, Fassier and Glorieux quoted the original classification as proposed by Sillence. ${ }^{[1]}$ This claassification had four types and has been increased to include at least seven types, based on clinical and radiographic findings. ${ }^{[3]}$ Type I is the mildest form, presenting with occasional fractures before puberty, minimal deformity and stature appearing normal. Type II is the most severe and is invariably lethal in the antenatal or immediate postnatal period. Uncalcified craniums, short limbs and narrow thoracic cavities are some of the features. Type III is the most severe type in childhood, presenting with frequent fractures leading to severe long bone deformities. It is also characterised by short stature. It is very disabling but is not lethal. It has traditionally been considered to be autosomal recessive or due to a new dominant mutation. ${ }^{[2]}$ Type IV is a mild form of this bone disease and presents with variable signs. The blue discolouration of the sclera is not apparent after childhood. Type $\mathrm{V}$ is moderately deforming, presenting with mild to short stature, dislocation of the radial head, mineralised interosseous membrane, hyperplastic callus formation, white sclera and no dentinogenesis imperfecta. Type VI is moderately to severely deforming, presenting with moderately short limbs, scoliosis, accumulation of osteoid in bone tissue, fish scale patterns of bone lammelation, white sclera and no dentinogenesis imperfecta. Type VII is moderately deforming, presenting with a short stature, short humeri and femora, coxa vara, white sclera and no dentinogenesis imperfect. Types I - IV are due to mutations in collagen genes, but mutations in these genes have been excluded in the other types. ${ }^{[1,3]}$

Beighton estimated the prevalence of OI type III in the black population of South Africa (SA) at 1:125 000 to $1: 200000{ }^{[4,5]}$ Other estimates state that for all types, the prevalence is between 1:10 000 to $1: 20000 .^{[6]}$

Until recently, treatment of fractures by means of orthopaedic management, which involves immobilisation and rodding, was the mainstay of patient care. ${ }^{[1,6]}$ Numerous medical treatments to reduce the fracture incidence including vitamin $\mathrm{D}$, fluoride, anabolic steroids, magnesium oxide, calcitonin and growth hormone have been attempted. None of these have been effective in reversing the underlying pathophysiology nor resulted in a clinical improvement. ${ }^{[3,6-8]}$ The aim of pamidronate treatment is to minimise fractures and maximise function. Bisphosphonate compounds, including pamidronate disodium (Aredia marketed by Novartis, Switzerland) and zoledronic acid, have been shown to reduce the fracture incidence and morbidity in more severe types of OI. ${ }^{[1]}$ Zoledronic acid has been introduced as a treatment for OI after the completion of this study. ${ }^{[9,10]}$

Pamidronate disodium is a bisphosphonate and thus has its effect by inhibiting bone reabsorption. It normalises the calcium level in hypercalcaemia by inhibiting osteoclast activity. This medication is given as a slow intravenous injection and its side-effects, which are usually mild and self-limiting, are characterised by a drop in serum calcium levels, transient leucopenia, influenza-like symptoms and fever reactions. ${ }^{[1,11]}$

Numerous authors have reported this form of treatment to be beneficial, with the effects being characterised by decreased fractures, reduction in bone pain and improving the quality of life for the patients. ${ }^{[2,6,8,11]}$ However, the majority of research has been performed on Canadians, North American Indians and Europeans. Very little information regarding patient attitudes or subjective assessment could be found when searching PubMed, only an assessment of quality of life after administration of oral bisphosphonates. ${ }^{[12]}$ No data about pamidronate treatment in 
black SA patients has been published. The efficacy and safety profile in black South Africans appears to be similar to published data (BD Henderson, presented at the 12th SA Society of Human Genetics congress in March 2007).

\section{Objective}

This study intended to assess subjective data regarding OI and pamidronate therapy in black patients diagnosed with OI type III and treated at Universitas Private Hospital, Bloemfontein. This included the sideeffects experienced, impact on the quality of their lives and overall attitude towards the disorder and the value of treatment.

\section{Methods}

A descriptive study using a researcheradministered questionnaire was undertaken. Two researchers interviewed the same patient simultaneously. None of the interviewers were involved in administering the treatment. This study was performed in 2009 and 2010 by undergraduate $\mathrm{MB}$ ChB students at the University of the Free State (UFS) and formed part the curriculum.

The protocol was approved by the ethics committee of the Faculty of Health Sciences at UFS (study No. 15/09). Informed consent was obtained from the individual, a parent or legal guardian for children, and assent, where applicable, from children old enough to understand. A pilot study was performed to confirm feasibility of the study. These results were not included in the study.

The patients approached were receiving treatment or had previously received treatment according to the protocol recommended by Glorieux et al. ${ }^{[1,2,9,13]}$ This entailed a 3-day admission every 4 months for intravenous administration of pamidronate disodium.
Thirty patients were approached and 26 were interviewed during an admission for treatment or by telephone. The parents of children too young to comprehend or respond to the questionnaire were interviewed.

\section{Results}

A total of 30 patients were approached for inclusion in the study. Of these, 28 were currently on treatment. Four patients did not respond or declined participation. All patients were considered to have type III OI or Bruck syndrome. The patients were resident in the Free State $(73.1 \%)$ or surrounding areas (26.9\%). The age range was from 1.5 to 24 years with a mean of 10.5 years. The majority of patients were too young to be interviewed and 19/26 (73.1\%) interviews were conducted with parents. The interviews were conducted face-to-face in $57.7 \%$ of cases, while $42.3 \%$ were telephonic. The female to male ratio was 1.36 .

All of the patients were diagnosed with the disorder before the age of 10 years old. The majority (88.5\%) had no knowledge or experience of the disorder prior to diagnosis.

The impact of the disorder on personal lives is reflected by parents responding that they felt embarrassed and isolated (10/19) by the disorder. The children also experienced isolation (2/7) and considered themselves handicapped and different (5/7) to other children. Three of the patients and six of the parents had either not considered this or considered themselves as normal (3/7 and $6 / 19$, respectively); others just said they had learnt to live with OI.

Fractures and bone pain were the most common symptoms experienced by patients prior to initiation of pamidronate treatment. These and other symptoms are reflected in Fig. 1.

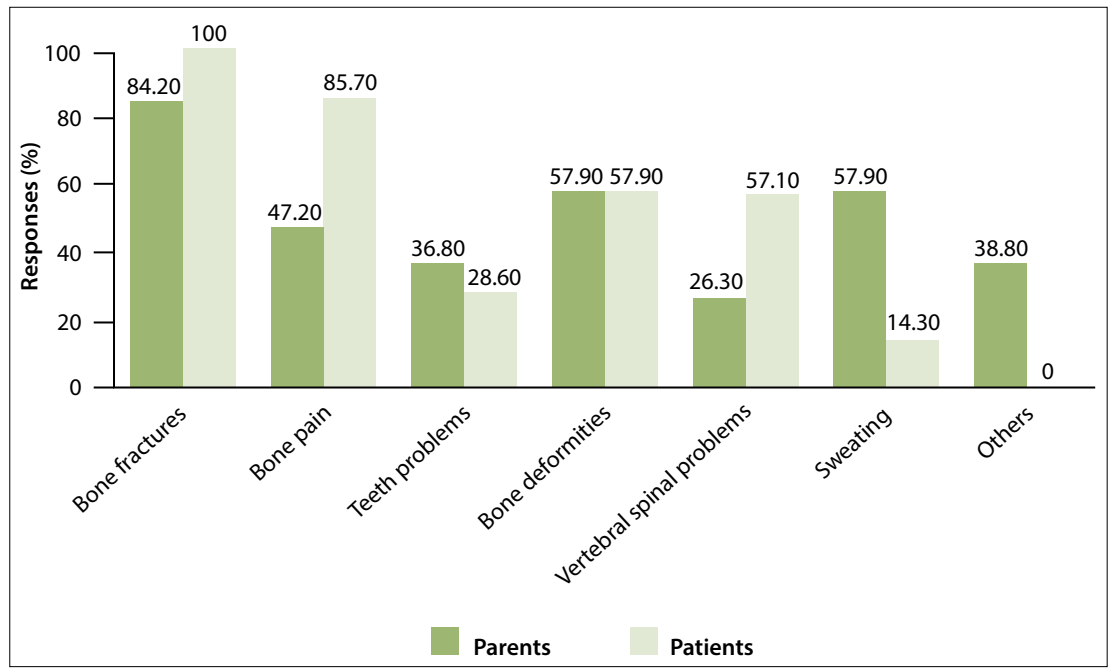

Fig. 1. Symptoms experienced before pamidronate treatment.
Two of the patients had used other medical treatment, namely calcium and vitamin $\mathrm{D}$, for management of the disorder and both indicated that pamidronate was better.

The siting of an intravenous line was not a problem for most of the participants with $73.7 \%$ of parents interviewed and $57.1 \%$ of patients interviewed tolerating this procedure well. For those participants who found the insertion of a line a problem, the reasons given were pain, discomfort and fear.

All participants felt that the treatment was beneficial and the reasons for this perception are reflected in Fig. 2. This figure clearly indicates that the participants felt that pamidronate had not only improved their physical wellbeing (fewer fractures, less pain and less sweating) but also their emotional wellbeing. A large percentage (73.1\%) of them felt stronger after receiving the treatment.

Side-effects were reported by $61.6 \%$ of respondents. Of those who experienced side-effects, fever and nausea were the most common and persisted for a few days (38.5\%). Side-effects persisted for a few hours in the rest of the patients who experienced any (23.1\%). The side-effects are listed in Table 1 . As can be noted, $57.9 \%$ of parents did not think their children experienced side-effects.

One of the two patients who had stopped treatment was contacted, and the reasons given for stopping treatment were unbearable side-effects and not knowing when the next admission was scheduled for.

Travelling costs and loss of income were not a significant concern for the participants with $73.1 \%$ indicating an out-of-pocket expense of less than R150 and 19.2\% experiencing loss of income.

All participants felt that a disability pension was justified and gave the following reasons: Disability makes it difficult to find employment with a reasonable salary and it is expensive to maintain a dignified lifestyle by a person living with a disability.

When asked for an overall opinion of the pamidronate treatment, $71.4 \%$ of parents interviewed and $68.4 \%$ of patients interviewed rated the treatment as excellent. All of the participants would recommend the treatment to other people. This is reflected in Fig. 3.

\section{Discussion}

These data indicate that black South Africans affected by OI experience similar symptoms and responses to the disorder as people elsewhere. ${ }^{[1,2,7,10,11]}$ The low number of participants reporting prior knowledge of the disorder supports the perception of an autosomal recessive or new dominant mutation as the underlying mechanism of disease in OI type III. ${ }^{[6]}$ 


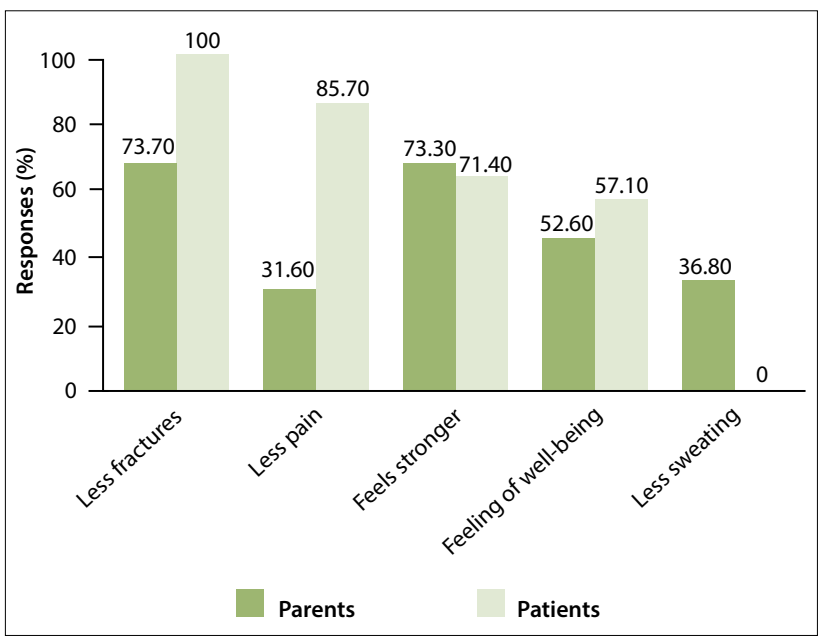

Fig. 2. Improvement after pamidronate treatment.

Table 1. Side-effects experienced

\begin{tabular}{lll}
\hline & $\begin{array}{l}\text { Parents' response } \\
(\mathbf{1 9 / 2 6 ) , \%}\end{array}$ & $\begin{array}{l}\text { Patients' response } \\
(\mathbf{7 / 2 6 )}, \%\end{array}$ \\
\hline Fever & 26.3 & 42.9 \\
Discomfort & 21.1 & 14.3 \\
Unease & 5.3 & 0.0 \\
Nausea & 10.5 & 42.9 \\
Headache & 10.5 & 28.6 \\
No side-effects & 57.9 & 28.6
\end{tabular}

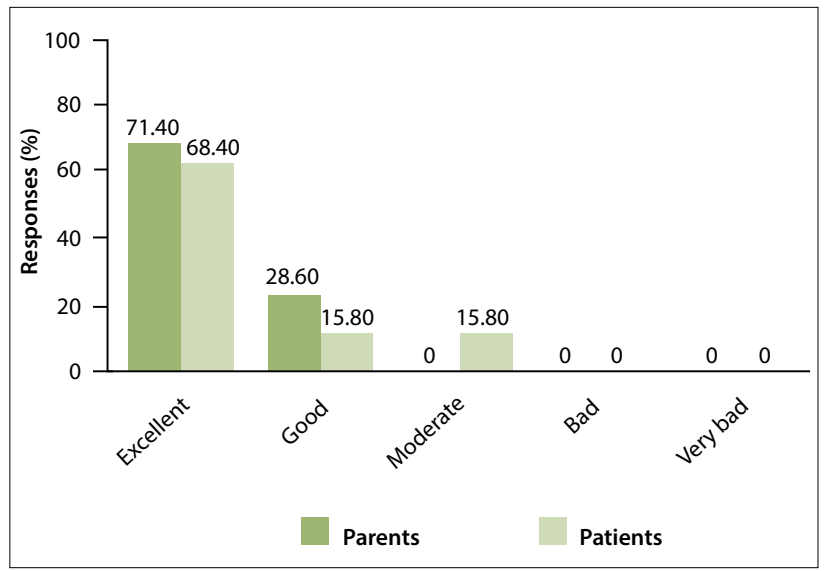

Fig. 3. Overall rating of pamidronate treatment.

Subjectively, pamidronate treatment is well tolerated and beneficial to this population group. The fact that so many rate the treatment as excellent must be considered in the light of this being the first medical treatment offered to alleviate the symptoms. The children and their parents perceive a benefit as demonstrated by the willingness to return for treatment every 4 months. The fact that an intravenous line was sited, and often replaced, for the duration of each 3-day hospital stay was not considered problematic must indicate that benefits outweigh this inconvenience. The perception of feeling stronger after the infusion would contribute to the positive attitude displayed by the participants. This positive attitude is supported by $100 \%$ of patients being willing to recommend this treatment to other people.
More side-effects were reported by children than parents. This may reflect the parents overlooking less striking side-effects such as nausea. The number of side-effects reported in this study is higher than other studies. The reason for this is unclear but may be due to the fact this study looked at collective recall of a number of treatment cycles whereas other studies collected data about side-effects during a specific treatment cycle.

One-third of participants had not considered themselves as different; however, all responded that a disability pension was indicated. This difference may reflect a general attitude to pensions in SA or reflect high acceptance of disability in the country. This should be investigated further.

The fact that many parents were interviewed on behalf of their children is a limitation to this study and may introduce a bias. It is doubtful that parents would expose their children and themselves to the inconvenience and financial strain of a 3-day hospital admission unless they perceived a benefit for their child. The low number of patients who do not continue receiving treatment would support that the parents perceived a benefit for their children but may have been a source of bias. For the majority of patients, pamidronate was the first medical treatment offered to them and would contribute to the high satisfaction experienced. The researchers intended that by having researchers not involved with the administration of the treatment, bias would be reduced and the participants would state their experiences without trying to please the treating physician.

\section{Conclusion}

The participants reflected a positive attitude towards pamidronate. The prolonged and frequent administration of the medication by an intravenous route was not troublesome to the patients. The side-effects were bearable and therefore the majority of patients were willing to return for every treatment cycle. The patients were satisfied with the improvement they experienced. This provides the first subjective support for using pamidronate in SA OI patients.

This study supports the use of bisphosphonates in young black patients with OI and demonstrates that it is well tolerated despite the route of administration. The impact of OI is no different in this study population in comparison with data from other populations. The parents seem to perceive side-effects differently to the children.

Acknowledgement. The authors would like to acknowledge and thank the Department of Biostatistics, UFS, for their assistance with the statistical analysis and also the participants for their friendly cooperation.

\section{References}

1. Zeitlin L, Fassier F, Glorieux FH. Modern approach to children with osteogenesis imperfecta. J Pediatr Orthop B 2003:12(2):77-87. DOI:10.1097/01.bpb.0000049567.52224. fa

2. Glorieux FH, Bishop NJ, Plotkin H, Chabot G, Lanoue G, Travers R. Cyclic administration of pamidronate in children with severe osteogenesis imperfecta. N Engl J Med 1998;339(14):947-952 DOI:10.1056/NEJM199810013391402

3. Rauch F, Glorieux FH. Osteogenesis imperfecta. Lancet 2004;363(9418):1377-1385

4. Beighton P, Versfeld G. On the paradoxically high relative prevalence of osteogenesis imperfecta type III in the Black population of South Africa. Clin Genet 1985;27:398-401. DOI:10.1016/S0140-6736(04)16051-0 Viljoen D, Beighton P. Osteogenesis imperfecta, type III: An ancient mutation in Africa? Am J Med Genet 1987;27(4):907-912.

6. Antoniazzi F, Mottes M, Fraschini P, Brunelli PC, Tatò L. Osteogenesis imperfecta: Practical treatment guidelines. Paediatr Drugs 2000;2(6):465-488.

7. Lee YS, Low SL, Lim LA, Loke KY. Cyclic pamidronate infusion improves bone mineralisation and reduces fracture incidence in osteogenesis imperfecta. Eur J Paediatr 2001;160(11):641-644.

8. Devogelaer J. New uses of bisphosphonates: Osteogenesis imperfecta. Curr Opin Pharmacol Devogelaer $\mathcal{~ J . ~ N e w ~}$

9. Devogelaer JP, Coppin C. Current treatment options and future prospects. Treat Endocrino 2006:5(4):229-242.

10. Vuorimies I, Toiviainen-Salo S, Hero M, Mkiie O. Zoledronic acid treatment in children with osteogenesis imperfecta. Horm Res Paediatr 2011;75(5):346-353. DOI:10.1159/000323368

11. Falk MJ, Heeger S, Lynch KA, et al. Intravenous bisphosphonate therapy in children with osteogenesis imperfecta. Pediatrics 2003;111(3):573-578.

12. Kok DHJ, Sakkers RJB, Castelein RM, Engelbert RHH. Quality of life in children with osteogenesis imperfecta treated with oral bisphosphonates (Olpadronate): A 2-year randomised placebo-controlled trial. Eur J Pediatr 2007;166(11):1155-1161. DOI:10.1007/s00431-006-0399-2

13. Cheung MS, Glorieux FH. Osteogenesis imperfecta: Update on presentation and management. Rer Endocr Metab Disord 2008:9(2):153-160. DOI:10.1007/s11154-008-9074-4 